\title{
NEAR AND FAR FIELD OPTICAL PATTERNS FORMATION BY ROTATIONAL SYMMETRY MASKS: APPLICATION TO OPTICAL SPATIAL SOLITON GENERATION
}

\author{
ANAHIT BADALYAN \\ Institute for Physical Research, National Academy of Sciences of Armenia, Ashtarak-2 \\ Ashtarak, 0203, Armenia \\ paytsar.mantashyan@gmail.com \\ PAYTSAR MANTASHYAN \\ Institute for Physical Research, National Academy of Sciences of Armenia, Ashtarak-2 \\ Ashtarak, 0203, Armenia \\ paytsar.mantashyan@gmail.com \\ VAHRAM MEKHITARYAN \\ Institute for Physical Research, National Academy of Sciences of Armenia, Ashtarak-2 \\ Ashtarak, 0203, Armenia \\ vm@ipr.sci.am \\ VARSENIK NERSESYAN \\ Russian-Armenian (Slavonic) University, 123 Hovsep Emin \\ Yerevan, 0051, Armenia \\ varsik91@gmail.com \\ RAFAEL DRAMPYAN \\ Institute for Physical Research, National Academy of Sciences of Armenia, Ashtarak-2 \\ Ashtarak, 0203, Armenia \\ Russian-Armenian (Slavonic) University, 123 Hovsep Emin \\ Yerevan, 0051, Armenia \\ rdramp@ipr.sci.am
}

In this report we present the formation of necklace type optical beams from Gaussian beam by rotational symmetry masks. The detailed studies of space evolution of the optical beam formation from the near field to the far field by the use of 7-fold symmetry mask and a single mode laser beam were performed. Different types of necklace beams with different numbers of "pearls" periodically or quasi-periodically situated along the different radii rings were observed during the space evolution of the optical diffraction pattern.

Keywords: Laser; diffraction; pattern formation; necklace type beams; optical soliton. 


\section{Introduction}

The existence and stability of 1D and 2D spatial optical solitons - self trapped optical beams of finite spatial cross-section that travel without the divergence, are the subject of increased interest due to the numerous possible applications, particularly, for controlling of optical beam propagation, in telecommunication systems, for all optical switching etc. Many properties of optical solitons that cannot be observed in bulk uniform nonlinear media have been discovered in nonlinear planar waveguide ${ }^{1}$, in nonlinear medium with ring-shaped ${ }^{2}$ and square photonic lattices ${ }^{3}$. Another way to form the optical solitons in nonlinear medium is the use of necklace ring beams with periodically modulated intensity along the azimuthal direction ${ }^{4,5}$.

In this work the formation of necklace type optical beams from Gaussian beam by rotational symmetry masks is presented. The detailed studies of space evolution of optical pattern formation from the near field to the far field by the use of 7-fold symmetry mask were performed for the first time.

\section{Experiment}

\subsection{Preparation of Different Symmetry Masks and Testing}

From 1 to 10 -fold axial symmetry masks were generated by computer graphic technique ${ }^{6}$. The masks consisted of transparent holes periodically disposed along the equidistantly positioned concentric circles, surrounding the central hole. The number of holes on the $i$ th circle was equaled $M_{i}=j i$, where $i=1,2,3 \ldots$ is integer and $j$ is the symmetry order of the mask. The masks of reduced size $(0.6 \mathrm{~cm}$ in diameter $)$ with $i=100$ were printed by high resolution printer (3300 dpi) on the transparent film. Both the positive and negative masks were prepared. The prepared negative masks had 10-60 $\mu \mathrm{m}$ distances between $10 \mu \mathrm{m}$ transparent holes located on the opaque disk. The whole mask consisted from $\sim 35000$ holes disposed along the 100 hypothetical concentric circles.

The far field diffraction patterns from these masks were studied by both red $633 \mathrm{~nm}$ and green $532 \mathrm{~nm}$ single mode laser beams. The far field diffraction patterns from 2, 3, 4 and 7-fold symmetry masks obtained by green single mode laser beam were presented in $^{6}$. Here we present the results of the far field diffraction patters obtained with the use of 5, 6, and 9-fold symmetry masks, as well as the detailed studies of space evolution of diffraction pattern for 7-fold symmetry mask.

\subsection{Experimental set-up for observation of space evolution of diffraction pattern by the use of 7-fold symmetry mask}

For the observation of space evolution of optical pattern formation from the near field to the far field we used the following experimental set-up (Fig.1). The single mode $633 \mathrm{~nm}$ laser beam with $17.7 \mathrm{~mW}$ power was used to observe space evolution of diffraction 
pattern by the use of 7-fold symmetry mask. The laser beam was expanded by confocal lenses and illuminated the mask. CCD camera was placed on the table with the possibility of fine moving.

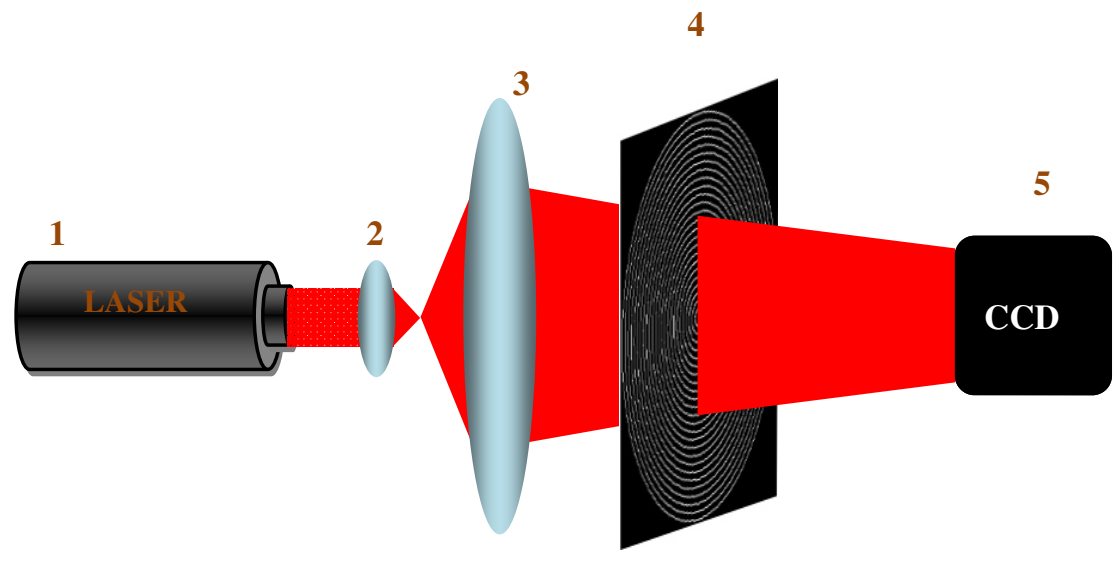

Fig.1. Experimental set-up: 1 -laser, 2,3 -lenses, 4- mask, 5- CCD camera.

Fig. 2 shows the fragment of 7 -fold rotational symmetry mask. Fig.3 a-d show examples of optical diffraction patterns obtained by red $633 \mathrm{~nm}$ single mode laser beam at different distances from the 7-fold symmetry mask.

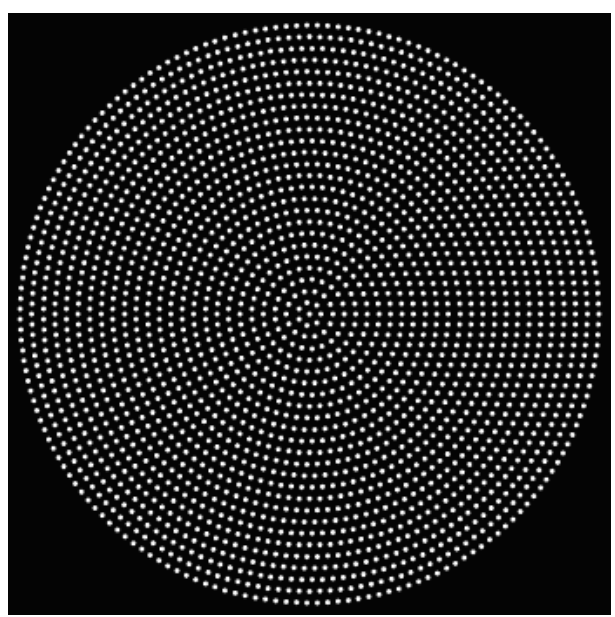

Fig.2. Fragment of 7-fold symmetry mask. 


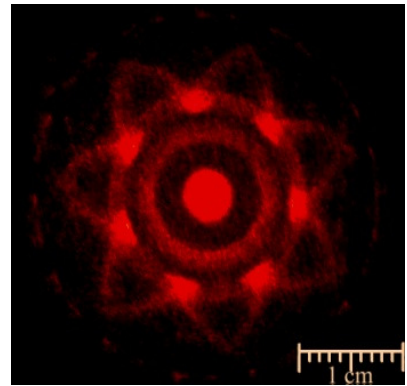

(a)

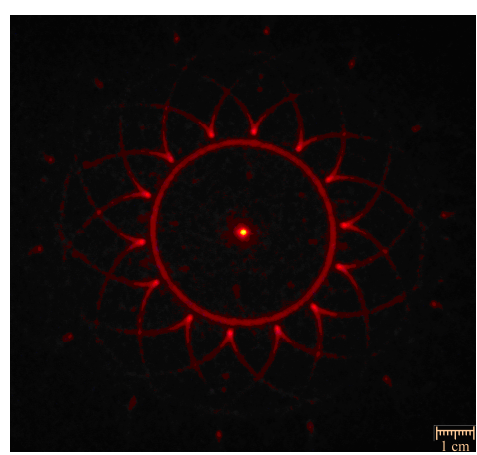

(c)

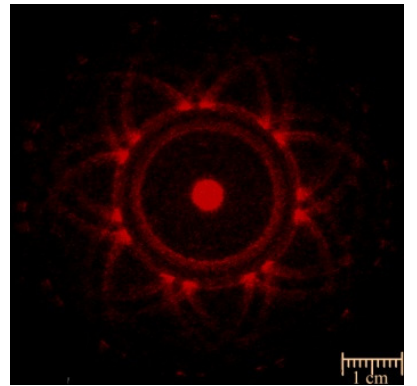

(b)

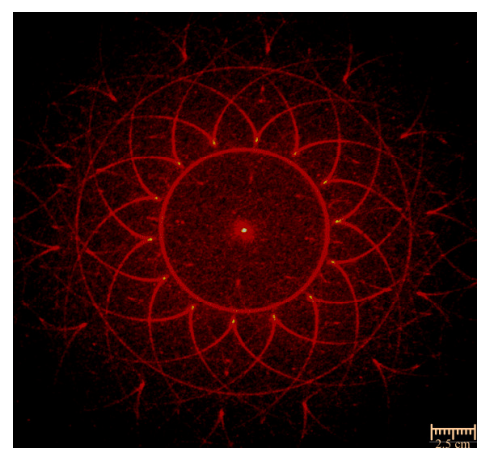

(d)

Fig.3. (a)-(d) Space evolution of diffraction pattern in the direction $\mathrm{Z}$ of beam propagation at different distances from the mask, $Z=43,63,113,243 \mathrm{~cm}$, respectively. The radii of the rings along which the "pearls" are disposed increase with the increase of the distance from the mask. The scale of $1 \mathrm{~cm}$ in the (a) is two times larger compared with (b), and four times larger compared with (c). The scale on (d) corresponds to $2.5 \mathrm{~cm}$.

Diffraction patterns show the evolution from 7 to 14 "pearl" necklace beams at the distances of 43, 63 and $113 \mathrm{~cm}$ Fig.3 (a-c), respectively. Fig.3a shows the formation of seven "pearl" necklace beam. Fig.3b shows the periodically disposed double "pearls" and Fig. 3c shows 14 "pearl" necklace beam. The radii of the rings along which the "pearls" are disposed increase with the increase of the distance from the mask. The diffraction pattern does not change further after distance of $243 \mathrm{~cm}$ from the mask in the far field Fig.3d.

\subsection{Formation of necklace-type beams by far-field diffraction from 5,6 and 9-fold symmetry masks}

Here we present the far field diffraction patterns obtained with the use of 5,6 and 9-fold rotational symmetry masks shown in Fig.4 (a-c). Fig.4d shows the far field diffraction 
pattern obtained from 5-fold symmetry negative masks by red $633 \mathrm{~nm} 17 \mathrm{~mW}$ single mode laser beam. Fig.4 e,f show the diffraction patterns obtained from 6 and 9-fold symmetry positive masks by a single mode green $532 \mathrm{~nm} 50 \mathrm{~mW}$ laser beam.

When the masks were illuminated by Gaussian beam the observations showed that for even symmetry masks the rotational symmetries of mask and far field diffraction pattern from the mask were the same (see Fig.4b,e). However, for odd symmetry masks the symmetry of diffraction pattern is twice as high compared with the the symmetry of the mask (Fig.2 and Fig.3 c,d, and Fig.4 a,d). The same results were obtained for 2,3 and 4-fold rotational symmetry masks ${ }^{6}$.

Fig.4d shows 10 "pearl" double ring necklace beam from 5-fold symmetry mask and Fig. 4f shows 18 "pearl" single ring necklace beam from 9-fold symmetry mask, whereas the Fig. 4 e shows 6-fold symmetry diffraction pattern from 6-fold rotational symmetry mask.

a

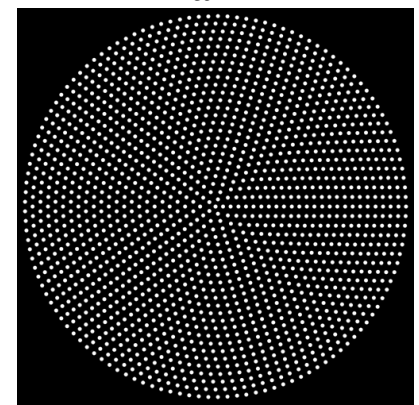

$\mathrm{J}=5$

d

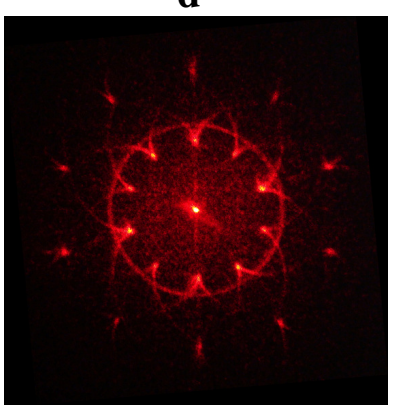

b

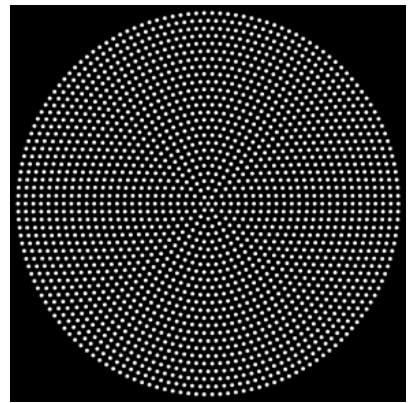

$\mathrm{J}=6$

e

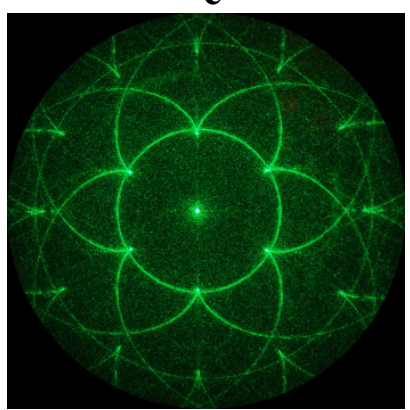

c

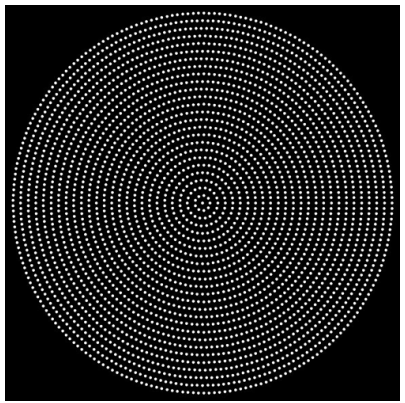

$\mathrm{J}=9$

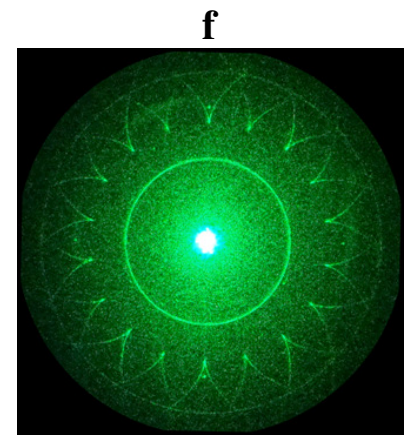

Fig. 4. Fragments of 5, 6 and 9-fold rotational symmetry masks (a,b,c), and far field diffraction patterns (d,e,f) obtained from 5, 6 and 9 fold symmetry masks, respectively, by red 633nm (d) and green $532 \mathrm{~nm}(\mathrm{e}, \mathrm{f})$ laser beams. The symbol $\mathrm{J}$ on the bottom of masks notes the rotational symmetry order of the masks. 


\section{Numerical simulations of diffraction patterns from rotational symmetry masks}

Numerical simulations of diffraction patterns from different symmetry masks were performed. Fig.5a shows the result of simulations of diffraction pattern from 6-fold symmetry mask (Fig.4b). The simulations were performed taking into account the mutual interference between beams diffracted from each hole. For comparison the experimentally obtained diffraction pattern from 6-fold symmetry mask by red $17 \mathrm{~mW}$ laser beam also is shown in Fig. 5b. The comparison between experimental results and simulations shows the perfect coincidence.
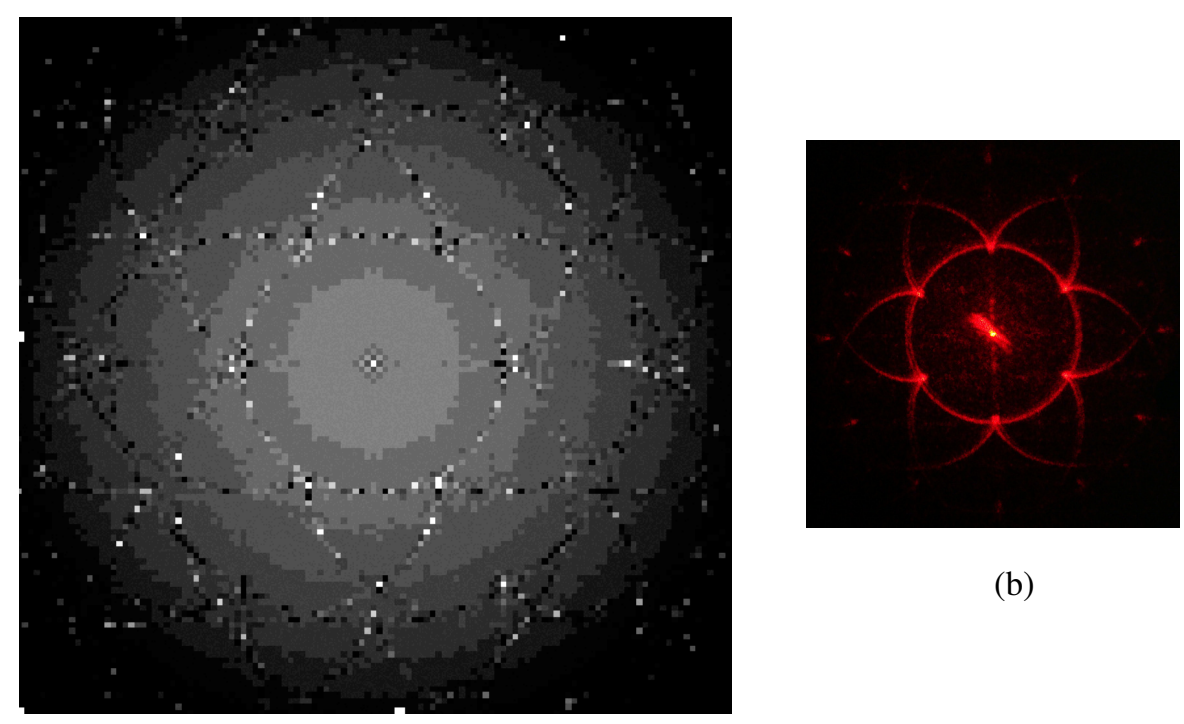

(b)

(a)

Fig. 5. (a) Results of numerical simulations of far field diffraction pattern from 6- fold rotational symmetry mask, the fragment of which is shown in Fig. 4(b). (b) Experimentally obtained far field diffraction pattern from 6-fold symmetry mask by red laser beam.

The simulations of space evolution of diffraction patterns from different rotational symmetry masks are under way and will be published elsewhere.

\section{Conclusions}

The formation of necklace type optical beams from Gaussian beam by rotational symmetry masks was observed. The detailed studies of space evolution of optical patterns formation from the near field to the far field by the use of 7-fold symmetry mask were performed and showed the formation of different types of necklace beams with different 
numbers of "pearls" periodically or quasi-periodically situated along the different radii rings.

The far field necklace type beam patterns from 5, 6 and 9-fold rotational symmetry masks were also observed. The numerical simulation of the far field diffraction pattern from 6-fold rotational symmetry mask was performed and showed perfect coincidence with experimental results.

The necklace beams can be used for the formation of spatial optical solitons in nonlinear solid crystals, such as lithium niobate, lithium tantalate, strontium barium niobate, in liquid crystals and in resonant atomic media.

\section{Acknowledgments}

The work was supported by International Science and Technology Center Grant, Project A - 1517.

\section{References}

1. S. Maneuf, R. Desailly, C. Froehly, Stable self-trapping of laser beam: observation in a nonlinear planar waveguide, Opt. Commun. 65, 193 (1988)

2. X. Wang, Zh. Chen, P. Kevrekidis, Observation of discrete solitons and soliton rotation in optically induced periodic ring lattices, Phys. Rev. Lett, 96, 083904 (2006).

3. J. Fleischer, M. Segev, N. Efremidis, D. Christodoulides, Observation of two-dimensional discrete solitons in optically induced nonlinear photonic lattices, Nature, 422, 147 (2003).

4. M. Soljacic, S. Sears and M. Segev, Self-trapping of "necklace" beam in self-focusing Kerr media, Phys. Rev. Lett. 81, 4851(1998).;

5. T. Grow, A. Ishaaya, L. T. Vuong, A. L. Gaeta, Collapse and stability of necklace beam in Kerr media, Phys. Rev. Lett. 99, 133902 (2007).

6. A. Badalyan, R. Hovsepyan, V. Mekhitaryan, P. Mantashyan, R. Drampyan, Combined interferometric-mask method for creation of micro- and sub-micrometric scale 3D structures in photorefractive materials, in International Conference on Laser Physics 2010, edited by Aram V. Papoyan, Proceedings of SPIE Vol.7998 (SPIE Belling-ham, WA, 2011) 7998OH-1-10. 